\title{
Zoledronic Acid for prevention of bone and muscle loss after BAriatric Surgery (ZABAS) - A study protocol for a randomized controlled trial
}

\section{Søren Gam ( $\sim$ sorengam1987@gmail.com )}

University of Southern denmark https://orcid.org/0000-0002-2461-2824

\section{Bibi Gram}

University hospital of Southern Denmark

Claus Bogh Juhl

University hospital of Southern Denmark

Anne Pernille Hermann

Odense University Hospital

Stinus Gadegaard Hansen

University hospital of Southern Denmark

\section{Research Article}

Keywords: Bariatric surgery, Gastric bypass, Sleeve gastrectomy, Bone loss, Muscle loss, Zoledronic Acid, Bone microarchitecture, Volumetric bone density, Lean body mass, Muscle mechanical function

Posted Date: February 4th, 2022

DOI: https://doi.org/10.21203/rs.3.rs-1188383/v1

License: (c) (1) This work is licensed under a Creative Commons Attribution 4.0 International License. Read Full License 


\section{Abstract}

\section{Background}

Bariatric surgery has adverse effects in the muscular-skeletal system with loss of bone mass and muscle mass and an increase in the risk of fracture. Zoledronic acid is widely used in osteoporosis and prevents bone loss and fracture. Bisphosphonates may also have positive effects in skeletal muscle. The aim of this study is to investigate the effects of zoledronic acid for the prevention of bone and muscle loss after bariatric surgery.

\section{Methods/design}

This is a randomized double-blind placebo-controlled study. Sixty women and men with obesity aged 35 years or older will complete baseline assessments before randomization to either zoledronic acid (5 mg in $100 \mathrm{ml}$ isotonic saline) or placebo (100 ml isotonic saline only) three weeks before surgery with Roux-enY-gastric bypass (RYGB) or sleeve gastrectomy (SG). Follow-up assessments are performed 12 and 24 months after surgery. The primary outcome is changes in lumbar spine volumetric bone mineral density (VBMD) assessed by quantitative computed tomography (QCT). Secondary bone outcomes are changes

in proximal femur VBMD assessed by QCT. Changes in cortical and trabecular bone microarchitecture and estimated bone strength will be assessed by high-resolution peripheral quantitative computed tomography (HR-pQCT). Cortical material bone strength at the mid-tibia diaphysis will be assessed using microindentation and fasting blood samples will be obtained to assess biochemical markers of bone turnover and calcium metabolism.

Secondary muscle outcomes include whole body lean mass assessed using dual-energy X-ray absorptiometry. Dynamometers will be used to assess handgrip, shoulder, ankle, and knee muscle strength. Short Physical Performance Battery, 7.6-meter walking tests, 2-minute walking test and a stair climb test will be assessed as biomarkers of physical function. Self-reported physical activity level is assessed using International Physical Activity Questionnaire (IPAQ).

Discussion

Results from this study will be instrumental for the evidence-based care of patients undergoing bariatric surgery.

Trial registration

ClinicalTrials.gov, ID: NCT04742010. Registered February $5^{\text {th }} 2021$.

\section{Background}

\section{Introduction}


Obesity, defined as a body mass index (BMI) $>30 \mathrm{~kg} / \mathrm{m} 2$ affects an increasing number of individuals worldwide and is associated with cardiovascular disease, diabetes mellitus and increased mortality (1). During several decades, bariatric surgery has been increasingly used to treat people with severe obesity especially using the Roux-en-Y gastric bypass (RYGB) procedure, with sleeve gastrectomy (SG) becoming increasingly popular in recent years (2). Bariatric surgery has, however, adverse effects in the muscularskeletal system as reflected by decreasing bone and muscle mass (3-7).

The loss of weight after bariatric surgery is mainly achieved within the first 6-12 months post-surgery and typically, weight is stable or slightly increasing afterwards. Despite weight stability, the age-related decline in bone mineral density (BMD) is accelerated, bone turnover remains increased and bone microarchitecture deteriorates which cause a reduction in estimated bone strength in the years following surgery $(3,6,7)$. This happens in parallel with an average of $25 \%$ decline in body lean mass two years after surgery and reduction in absolute muscle strength $(4,5)$. Altogether, this may cause a higher risk of falls and a reduction in bone strength that may both contribute to an increase in fracture risk. Several studies have documented that people undergoing bariatric surgery have an increased risk of fracture (811) both compared to individuals with similar age, sex and BMI and the general population and this risk ratio seems to increase further with time from surgery (10-12).

The negative skeletal effects of bariatric surgery are presumably multifactorial and mechanisms may involve nutritional factors, mechanical unloading, hormonal factors, and changes in body composition and bone marrow fat (13). After bariatric surgery and RYGB in particular, the gastrointestinal absorption of calcium and Vitamin $D$ is compromised which causes secondary hyperparathyroidism and an increase in bone remodeling (14). Compromised absorption of nutrients and skeletal unloading, however, do not fully account for the increase in bone resorption, and changes in hormones related to adipose tissue and glucose homeostasis hormones may also be of importance. Furthermore, major changes are observed in the secretion of gastrointestinal hormones such as serum peptide YY, gastric inhibitory protein (GIP) and glucagon-like-peptide 1 and 2 (GLP1 and GLP2) that have been shown to modulate bone remodeling (13).

Following bariatric surgery patients are routinely advised supplements with calcium and Vitamin D. Also, to preserve muscular-skeletal health after surgery, nutrition or exercise regimens or both in combination have been tested to prevent the loss of bone and of muscle mass and muscle strength after bariatric surgery $(4,5,15)$, although with limited success. Therefore, interventions that can preserve muscularskeletal health when undergoing bariatric surgery are warranted.

The bisphosphonate zoledronic acid is widely used for prevention of bone loss and fracture in women and men with osteoporosis $(16,17)$. It acts through inhibition of osteoclasts. It is administered once yearly although effects in most cases last up to two to three years after administration (18). The use of bisphosphonates in a setting of bariatric surgery is unclarified. Two ongoing randomized clinical trials (NCT03411902 and NCT0427939) that use bisphosphonates are also aiming to clarify this matter. 
Preliminary results in a sample size of four showed that zoledronic acid may preserve trabecular bone density in the spine 24 weeks post-surgery but was not able to prevent bone loss in the hip (19).

In addition to preserving bone mass, animal- and small scale human studies indicate that bisphosphonates may also be able to preserve muscle mass (20-22) and improve muscle strength and physical performance $(23,24)$. Bisphosphonates may prevent muscle wasting by downregulating the osteoclast activity and thereby lower the release of cytokines like TGF- $\beta$ that are embedded in the bone matrix. These cytokines have a catabolic effect on muscles (25). Moreover, bisphosphonates are also reported to reduce pain and improve activities of daily living in postmenopausal women with existing vertebral fractures (26), which may lead to increased physical activity and subsequently preserve muscle mass.

In this randomized placebo-controlled study, we aim to assess effects and safety of zoledronic acid on surrogate markers of bone strength in patients undergoing bariatric surgery. Also, effects on muscle mass and function will be assessed. We hypothesize that treatment with zoledronic acid will prevent the increase in bone turnover and preserve surrogates of bone strength in patients undergoing bariatric surgery. Additionally, we postulate that zoledronic acid will antagonize the post-surgery loss of muscle mass and function.

\section{Study objectives}

To evaluate the effects of zoledronic acid on 1) bone mineral density, structure, material quality and metabolism and 2) muscle mass, muscle strength, physical function and physical activity in patients after bariatric surgery.

\section{Methods/design}

\section{General design}

This is a single center randomized double-blinded, placebo-controlled study conducted at the University hospital of Southern Denmark. The study will be conducted in accordance with the Standard Protocol Items: Recommendations for Interventional Trials (SPIRIT) 2013 Checklist for RCTs (Additional file 1) (27) and the Declaration of Helsinki, approved by the Regional Committee on Health Research Ethics for Southern Denmark (project identifier S-20190134), Danish Medicine Agency (protocol number: ZOL6700), and registered at ClinicalTrials.gov (NCT04742010) and EudraCT (2019-001650-26). In case protocol modifications are required during the trial these will be reported to the relevant authorities. Enrolment began February 2021 and is expected to be completed approximately May 2022. The results will be reported in accordance with the CONSORT statement for trial reports and results, positive or negative, will be disseminated. 
Figure 1 shows the design of the study. After enrollment, the subjects complete baseline assessments before randomization to either zoledronic acid or placebo. The subject will receive zoledronic acid or placebo -59 to -7 days prior to surgery. Follow-up assessment will be performed 12 and 24 months after surgery. For the assessments at each time point, the subjects will visit the hospital at two occasions. At day 1 , the following assessment will be conducted in the order: overnight fasting blood samples; Quantitative Computer Tomography (QCT); dual-energy X-ray absorptiometry (DXA); muscle function; physical function; physical activity and for day 2: high-resolution peripheral quantitative computed tomography (HR-pQCT); microindentation. Prior to day 1, the subject will be instructed to refrain from any strenuous physical activity and alcohol $>24$ hours before assessments.

\section{Subjects and recruitment}

Patients referred for bariatric surgery (RYBG or SG) are invited to participate in the study. As part of their routine clinical visits, patients will be asked for potential interest for participating and if agreed written information will be mailed. A member of the study team will then contact the patients. After the oral information, the patient is given time to consider participation and afterwards the informed consent is obtained.

\section{Inclusion criteria}

Patients that are $\geq 35$ years old and are eligible for bariatric surgery according to Danish National Guidelines will be included in the study. These include either $1: \mathrm{BMI} \geq 35 \mathrm{~kg} / \mathrm{m}^{2}$ and an obesity related comorbid condition of type 2 diabetes, sleep apnea, arthrosis of the hip or knee, polycystic ovary syndrome with an unmet wish for pregnancy or treatment resistant hypertension, or 2 : BMI $\geq 40 \mathrm{~kg} / \mathrm{m}^{2}$ with an obesity related health risk other than those specified in 1.

\section{Exclusion criteria}

Patients were excluded if they had a history of medical disorders with known effects on bone metabolism (hypo- and hyperthyroidism are allowed if treated and stable) or metabolic bone disease (osteoporosis is allowed). Patients with previously treatment with anti-osteoporotic agents, current treatment with oral glucocorticoids or other drugs with effects on bone metabolism (inhaled and topic glucocorticoids, antiepileptic agents, anticonceptives or other agents with estradiol are allowed) were excluded. Other exclusion criteria were pregnancy or breastfeeding, chronic kidney disease with estimated GFR $<45$ $\mathrm{ml} / \mathrm{min}$., hypocalcemia, hypersensitivity to bisphosphonates, mannitol or sodium citrate. Fertile women that participate in this study must accept to use contraception with intrauterine devices or oral contraceptive pills with estradiol) until one year after the administration of zoledronic acid/placebo. In addition, before the administration of the study drug, all fertile women will be screened for pregnancy using a urine human chorionic gonadotropin (HCG) test. 


\section{Bariatric surgery}

Routine bariatric surgery (RYGB or SG) will be performed within an interval of 30 days and 8 months after study inclusion. Endoscopic surgery will be performed by one of three skilled surgeons. The RYGB procedure includes a reduction of the gastric pouch to $20-30 \mathrm{~mL}$, a $60 \mathrm{~cm}$ bilio-pancreatic limb and a Roux limb of $150 \mathrm{~cm}$. The SG procedure includes a gastric-volume reduction of 75 to $80 \%$ by resecting the stomach alongside a 30 -French endoscope beginning $3 \mathrm{~cm}$ from the pylorus and ending at the angle of His.

\section{Supplements with calcium and Vitamin D}

According to Danish guidelines all subjects will be advised supplements with calcium (citrate or carbonate) $400 \mathrm{mg}$ two times daily and Vitamin D 38 microgram daily. Subjects will be advised to take supplements from inclusion and throughout the study. In case of serum 25-hydroxy-Vitamin D levels below $25 \mathrm{nmol} / \mathrm{l}$ a loading dose of 100.000 units Vitamin D3 will be given orally.

\section{Randomization}

After informed consent and baseline assessments, subjects will be randomized into two groups: zoledronic acid or placebo. The randomization sequence will be created using computer-generated software (28) stratifying patients with a 1:1 allocation using random block sizes of 2, 4 and 6 . A randomization code stratifying an equal number of subjects having RYGB or SG into each study arm will be applied. Subjects will be blinded during the whole study. After the acquisition of 12 months assessments, a non-member of the study team will prepare an un-blinded, anonymous data report for reporting of 12 months outcomes. All members of the study team remain blinded throughout the study.

\section{Intervention}

Subject's will receive a single dose of zoledronic acid $5 \mathrm{mg}$ or placebo 21 days before bariatric surgery (an interval of 59 to 7 days is accepted). Zoledronic acid or placebo will be administered in a solution containing $100 \mathrm{ml}$ normal saline and slowly infused intravenous ( $\geq 15$ minutes). Due to the risk of anaphylaxis, subjects are observed at least 30 minutes on the study site after the infusion. The Pharmacy, University hospital of Southern Denmark will prepare the study medicine on the day of administration. Preparation is performed at a separate location in the hospital away from the research facility. Zoledronic acid or placebo solutions will be identical and labeled only with the randomization number of the particular subject.

\section{Endpoints}


All outcomes will be assessed at baseline and 12 and 24 months after surgery. Table 1 shows a brief overview of primary and secondary endpoints in the study.

\section{Primary endpoints}

The primary endpoint is percentage change in volumetric BMD (VBMD) at the lumbar spine (L1-L2) measured by a non-contrast enhanced abdominal/pelvic Quantitative Computed Tomography (QCT) (Siemens SOMATOM FORCE; Siemens Healthcare AG, Erlangen, Germany). The obtained images will be transferred to a QCT workstation and analyzed using Mindways QCT pro software (Mindways Software Inc., Austin, TX, USA). QCT is a multi-planar, three-dimensional bone densitometry imaging device and is chosen as the primary outcome since QCT provides detailed and accurate descriptions and analysis of bone shape, size and achitecture at macroscopic level, which is in contrast to DXA that only provides information about areal BMD (aBMD) (29). 


\begin{tabular}{|c|c|c|c|}
\hline & Measurement & Site & Time (months) \\
\hline \multicolumn{4}{|l|}{$\begin{array}{l}\text { Primary endpoints } \\
\end{array}$} \\
\hline vBMD & QCT & Lumbar spine (L1 -L2) & 12 \\
\hline \multicolumn{4}{|l|}{ Secondary Endpoints } \\
\hline vBMD & QCT & Lumbar spine (L1 -L2) & 24 \\
\hline vBMD & QCT & Proximal femur & 12 and 24 \\
\hline aBMD & DXA & $\begin{array}{l}\text { Hip } \\
\text { Lumbar spine }\end{array}$ & 12 and 24 \\
\hline Lean and fat mass & DXA & Whole body & 12 and 24 \\
\hline Bone microarchitecture & HR-pQCT & $\begin{array}{l}\text { Distal Radius } \\
\text { Distal Tibia }\end{array}$ & 12 and 24 \\
\hline Cortical bone strength & Microindentation & Mid Tibia & 12 and 24 \\
\hline $\begin{array}{l}\text { Biochemical markers of bone } \\
\text { remodeling }\end{array}$ & $\begin{array}{l}\text { CTX1 } \\
\text { P1NP }\end{array}$ & Blood & 12 and 24 \\
\hline Muscle strength & Dynamometer & $\begin{array}{l}\text { Handgrip } \\
\text { Knee extension/flexion } \\
\text { Foot dorsi/plantar flexion } \\
\text { Shoulder elevation }\end{array}$ & 12 and 24 \\
\hline Physical function & $\begin{array}{l}\text { SPPB } \\
\text { T25FWT } \\
\text { 2MWT } \\
\text { Stair climb }\end{array}$ & & 12 and 24 \\
\hline Physical activity & IPAQ & & 12 and 24 \\
\hline
\end{tabular}

Time is measured in months; QCT Quantitative Computed Tomography; DXA whole-body dual-energy X-ray absorptiometry; HR-pQCT high-resolution peripheral quantitative computed tomography, $C T X-1$ carboxyterminal type-1 collagen; P1NP Procollagen type-1 amino-terminal propeptide; SPPB Short Physical Performance Battery; T25FWT timed 25-ft walk test; $2 M W T 2$ minute walk test; IPAQ The International Physical Activity Questionnaire

\section{Secondary endpoints}

\section{Anthropometrics and body composition}

Fat and lean mass will be assessed by a whole-body dual-energy X-ray absorptiometry (DXA) scan (Hologic Horizon A; Waltham, MA, US). DXA is a low resolution, uniplanar, two-dimensional bone densitometry imaging device that assesses mass quantities and densities for both full-body and segmental projections. DXA is chosen due to its ability to effectively differentiate hard tissue from soft tissue, and fat mass from lean mass. 
Height and weight will be measured by wall mounted stadiometer and weight scales (Seca 899, SECA $\mathrm{GmbH}$, Hamburg, Germany). Waist circumference will be measured in the middle of the distance between the $12^{\text {th }}$ rib and the anterior-superior iliac spine while keeping the measuring tape horizontally. Hip circumference will be measured across the widest part of the hips with the measuring tape horizontally.

\section{Bone mass}

Changes in wbBMC, aBMD of the hip (total hip region) and lumbar spine (L1-L4) will be measured by DXA.

\section{Bone microarchitecture}

Assessment of bone geometry, vBMD, cortical and trabecular microarchitecture and estimated bone strength at the distal radius and tibia will be performed using high-resolution peripheral quantitative computed tomography (HR-pQCT) (XtremeCT II, Scanco Medical AG, Brutisellen, Switzerland). HR-pQCT provides information at the microscopic level about cortical and trabecular microarchitecture (29). The reported outcomes will be compartmental geometry, vBMD, bone volume per trabecular volume (BV/TV), trabecular number, trabecular thickness, trabecular separation, cortical thickness, cortical porosity, and finite element analysis estimates of biomechanical competences. A detailed description of the scanning protocol and the image analyses have previously been published (30-32).

\section{Cortical bone strength}

Assessment of cortical Bone Material Strength index (BMSi) at the mid-tibia will be performed using impact microindentation (The OsteoProbe, Active Life Scientific, California, USA). BMSi is a marker of bone stiffness. Microindentation is a minimally invasive procedure with minimal experienced pain and does not affect the ability of the individual to walk immediately afterwards. Briefly, the microindentation procedure consist of three steps: 1) subcutaneous local anesthesia of the mid-tibia region, 2) penetrating the skin and periosteum with the probe until it is reaching the bone cortex. A total of ten indentations are performed with the probe positioned perpendicular to the bone surface, 3) last, a single indentation in a reference material (the BMSi-100 Reference Material (polymethyl methacrylate)) is performed. BMSi is defined by the ratio of the penetration of the probe into the bone compared to a reference standard material and expressed in absolute units. A detailed description the procedure is found elsewhere (33).

\section{Biochemical calcium metabolic markers}

Markers of bone formation (procollagen type-1 amino-terminal propeptide (P1NP)) and bone resorption (carboxy-terminal type-1 collagen (CTX-1)) as well as calcium, parathyroid hormone and 25-hydroxy Vitamin $\mathrm{D}$ will be measured using standardized equipment. Blood samples will be taken after an overnight fast and stored a $-70^{\circ} \mathrm{C}$ in a biobank until analyses.

\section{Muscle strength}


Dynamic and isometric knee flexor/extensor (KF/KE) and ankle dorsi/plantar flexor (DF/PF) strength of the non-dominant leg will be measured in an isokinetic dynamometer (Biodex, System 4, Biodex Medical System Inc, New York, USA). The order of assessment will be dynamic contraction prior to isometric, and $\mathrm{KF} / \mathrm{KE}$ prior to DF/PF. For the dynamic contractions, the subject will perform three attempts separated by 30 seconds rest in between. The angular velocities will be $180 \%$ s and $90 \%$ for KF/KE and DF/PF respectively. For the isometric contractions, the subjects will perform three to five attempts lasting three seconds with 60 seconds rest in between. KF and KE will be performed in fixed positions of $30^{\circ}$ and $75^{\circ}$ of the knee angle and for $\mathrm{DF}$ and $\mathrm{PF}$ will be performed in $20^{\circ} \mathrm{PF}$ and $0^{\circ} \mathrm{PF}$, respectively. For all dynamic and isometric attempts, the subjects will be instructed to relax, and after an auditory signal attempt to contract as "hard and fast" as possible. The reported outcomes will be muscle force normalized to body mass $\left(\mathrm{Nm} \cdot \mathrm{Kg}^{-1}\right)$.

Maximal shoulder elevation strength will be measured with Bofors MODEL dynamometer (Bofors MODEL dynamometer, Bofors Elektronic, Karlskoga, Sweden) mounted in a reproducible standardized setup (4). The subjects perform 3-5 maximal isometric shoulder elevation in a seated position with 60 seconds rest in between.

Maximal handgrip strength (HGS) of the non-dominant hand will be measured by handgrip dynamometer (Jamar Plus, Patterson Medical, Warrenville, IL, US). HGS measurements will be conducted in accordance to recommendation from the American Society of Hand Therapists (34).

\section{Physical function}

Short Physical Performance Battery is a set of tests that determine mobility and function in some daily living activities, such as balance, walking speed and strength. Briefly, SPPB has been validated to assess lower extremity function and balance in older adults (35). SPPB includes three balance tests that increases in difficulty: parallel-, semi tandem- and tandem stand. Walking speed is assessed by performing two trials of 3-meter walks at the subject's usual pace. Leg strength will be assessed by a chair stand test with five repeats performed as quickly as possible. SPPB will be scored according to guidelines (35).

In addition, a short walking test (T25FWT) will be used to assess the subject's fast walking capacity and ability to initiate propulsive movement. Subjects will perform two $25-\mathrm{ft}(7.6 \mathrm{~m})$ walks as fast as possible from standing position. The fastest attempt will be selected for further analysis and is reported as velocity $\left(\mathrm{m} \cdot \mathrm{s}^{-1}\right)$ and time (s).

A 2-minute walking test (2MWT) will be used to assess walking endurance capacity. The subject's is instructed to walk as many rounds as possible on a 20-m track for 2 minutes. 2MWT will be reported as velocity $(m \cdot s-1)$ and distance $(m)$.

A 9-step stair (depth $28.5 \mathrm{~cm}$, height $17 \mathrm{~cm}$ ) ascend test will be used to assess the subject's stair climbing abilities and functional power ((body weight · gravity $\left(9.81 \mathrm{~N} \cdot \mathrm{kg}^{-1}\right) \cdot$ stair height $(1.53 \mathrm{~m}) /$ time). The 
subjects will be instructed to ascend 9-steps one step at a time as fast as possible. The subjects are allowed to use assistive devices and the stair railing if needed. Two attempts are performed, and the fastest attempt will be selected for further analysis and be reported as velocity $\left(\mathrm{m} \cdot \mathrm{s}^{-1}\right)$, time $(\mathrm{s})$ and power (watt).

\section{Physical activity}

Changes in physical activity and exercise habits during the study may impact bone and muscle outcomes. To assess potential confounding, the physical activity and exercise habits will be assessed using the International Physical Activity Questionnaire (IPAQ). Physical activity will be assessed in at baseline, 12 and 24 months. The reported activities will be transformed to metabolic equivalent/week and the subjects are categorized as having a low, moderate, or high physical activity level according to guidelines (36). IPAQ is chosen since Ekelund et al. (36) have shown that the short, last 7-days version of the IPAQ has acceptable validity for assessing MET/week in adults.

\section{Safety}

Safety will be monitored in accordance with Good Clinical Practice Guidelines from the time of inclusion and until 12 months after surgery. All serious adverse events (SAE) and reactions (SAR) are reported to The Regional Committees on Health Research Ethics for Southern Denmark and The Danish Health and Medicines Authority. The GCP unit at Odense University Hospital will audit the study. At the 1- and 12months visits, subjects will be specifically asked regarding adverse events. In addition, subjects are informed to notify the study site in case of adverse events or reactions to ensure continuous registration and reporting. Four weeks after surgery blood samples are collected to assess occurrence of hypocalcemia and renal function. Fertile women that participate in the study accept to use intrauterine contraceptive devices or oral anticonception pills containing estrogen from inclusion and until one year after the administration of the study intervention. Screening for pregnancy using a urine HCG test is performed on the day of administration of the study intervention. Harms related participation in the study are covered by the hospital's insurance.

\section{Statistical analysis}

\section{Sample size determination}

The sample size estimation is based on the primary outcome change in lumbar spine vBMD during12 months after surgery. A decline from 194 to $182 \mathrm{mg} / \mathrm{cm} 3$ is assumed (mean values with SD of 27 $\mathrm{mg} / \mathrm{cm} 3)(17)$ in those receiving placebo while those receiving zoledronic acid are assumed to have unchanged BMD. Then a total of 21 subjects in each group will be sufficient to detect a statistically significant difference between the groups (repeated measures estimation, power 0.80 , alpha 0.05 , 
correlation 0.86). To allow for dropouts we aim to include 30 patients in each study arm. To ensure participant retention to the follow-up assessment, a study member will regularity be in contact with the participant by mail and phone throughout the whole study.

\section{Statistical methods and data managing}

Primary and secondary outcomes will be analyzed according to the intention to treat principle and subsequently per protocol. Missing data will be handled by multiple imputations, assuming missing values are randomly distributed. To assess the effects of the intervention, an age and sex-adjusted mixed effect model with repeated measures including a term for the interaction of group (zoledronic acid or placebo) and time will be used to assess changes from baseline at 12 and 24 months after surgery.

Study data are stored using REDCap (Version 9.1.15 - (c) 2020 Vanderbilt University, Tennessee, US), an electronic data capture tool hosted at the University of Southern Denmark. (37)

\section{Discussion}

This is the first study to assess the effects of zoledronic acid on bone and muscle outcomes after bariatric surgery. In a two-year randomized, double-blinded, placebo controlled study effects of zoledronic acid on surrogates markers of bone strength including bone mass, architecture and bone quality will be assessed. In addition, effects on muscle mass and strength and biomarkers of physical function are evaluated.

Although bariatric surgery has well documented positive effects on diabetes and cardiovascular disease, it has become clear that adverse reactions occur in the muscular-skeletal system. Clinical guidelines advise supplements with calcium and vitamin $D$ after bariatric surgery yet prospective studies with up to seven years of follow-up have documented ongoing bone loss despite such recommendations. In addition to calcium and vitamin $\mathrm{D}$, other non-pharmacological interventions have been suggested to prevent bone loss following bariatric surgery. For example, a two-year randomized controlled trial found that a regimen of high-dose vitamin $D$, calcium, high protein intake and a physical activity program was able to reduce but not prevent the negative impact on BMD and accelerated bone turnover (5). Similar results were reported following combined supervised aerobic and resistance training (38). After bariatric surgery, patients also experience a substantial decline in lean body mass assessed two years after surgery (5). While the overall physical activity may be improved in parallel with the loss of weight, absolute muscle strength declines after surgery (4). Gains in absolute muscle strength can be achieved following bariatric surgery with progressive moderate-intensity strength training with additional protein supplementation (15) or combined aerobic and strength training (38). However, combined aerobic and strength training or strength training in combination with protein supplementation was insufficient to a prevent muscle loss $(15,38)$. This loss of muscle strength may cause a higher risk of falls, which in combination with the reduction in bone strength, contributes to the increase in fracture risk. Therefore, interventions that can secure muscular-skeletal health after bariatric surgery are warranted. 
In postmenopausal osteoporosis, treatment with zoledronic acid reduces the risk of clinical vertebral fractures with approximately $75 \%$ and the fracture-reducing efficacy is generally well reflected in changes in bone mass during treatment (16). With the number of subjects and short duration in the current study a reduction in fracture occurrence is not expected; rather the aim is to document effects on bone mass and other surrogates of bone strength to make probable that bisphosphonates are also effective for preserving bone health in a setting of bariatric surgery. When used in osteoporosis, zoledronic acid is administered once yearly, however, a fracture-reducing efficacy has also been observed with less frequent administration (39). To assess if such longer-term effects are also seen in this setting, we chose to administer zoledronic acid once preoperatively while assessing outcomes after both 12 and 24 months.

The potential effects of bisphosphonates in muscle tissue may be attributed to the hormonal and mechanical interaction between the bones and muscles (24). In a setting of bariatric surgery where large declines in lean body mass are observed, we aim to explore this proposed effect of bisphosphonates on muscle outcomes including muscle strength, mass and physical function.

In general, obesity is related to high bone mass, likely a result of large mechanical demands on the skeleton given a high body weight. It has however been observed that bone strength in people with obesity may not fully adapt to body weight which may therefore lead to skeletal fragility $(40,41)$. Accordingly, a number of studies have documented an increased risk of fractures at some, but not all, skeletal sites including proximal humerus, upper leg and ankle fractures compared to normal weight individuals $(42,43)$. Therefore, in obesity, bone mass may not reflect the risk of fracture to the same extent as in normal-weight individuals. For this reason, we chose not to use bone mass as a selection criterion but rather to include patients aged 35 years or older. At this age peak bone mass has been obtained and therefore, we do not expect any further increments in bone mass during the study that could confound study findings.

We have chosen to include both RYGB and SG since both procedures induce similar reductions in bone mass in the short- (44-46) and intermediate-term (46) although some studies have shown some skeletal site-specific difference between the procedures (44). Stratifying an equal number of subjects having RYGB or SG into each study arm will take account for such site-specific differences.

Since effects of osteoporosis medications are unclarified in this setting, results from this study, positive or negative will be of importance for clinicians to offer evidence-based care. In case zoledronic acid antagonizes the bone metabolic imbalance induced by bariatric surgery, it will be of clinical relevance for both patients and clinicians. Should zoledronic acid enter clinical care in this setting, patients will easily be able to adhere to treatment given the administration once yearly. In addition, since the drug is no longer patented health care costs will be low. Furthermore, this study will explore potential effects of bisphosphonates in muscle tissue that will advance research in musculoskeletal pathophysiology and the proposed hormonal connections between muscle and bone.

\section{Limitations}


There are some limitations of this study. First, bone or muscle biopsies are not obtained. Although effects of zoledronic acid are well documented in postmenopausal osteoporosis using bone biopsies, it would have been of interest to examine potential effects of zoledronic acid in bone and skeletal muscle at the tissue and cellular levels in a setting of bariatric surgery. However, with the number of the examinations included in the study we chose not to obtain biopsies for logistic reasons and since these are invasive procedures that can lead to complications. In case effects of zoledronic acid are observed in the muscle outcomes this could be explored in a subsequent study. Second, bones and muscle are anabolic sensitive to mechanical loads such as physical activity and exercise (47). Therefore, differences in physical activity and exercise habits would potentially compromise the interpretation of the outcomes. Accelerometers can accurately assess physical activity. However, accelerometers require technical expertise, specialized hardware, software, and individual programming (48). Instead, IPAQ will be used to assess physical activity and exercise habits (36).

\section{Conclusion}

This two-year randomized, double-blinded, placebo-controlled study will assess effects of zoledronic acid on surrogate markers of bone strength people with severe obesity undergoing bariatric surgery. In addition, a potential effect of bisphosphonates in skeletal muscle is sought through assessments of muscle mass and strength and biomarkers of physical function.

\section{Declarations}

Trial registration

This trail was registered February $5^{\text {th }} 2021$ at ClinicalTrials.gov, ID: NCT04742010. The first subject was recruited February $11^{\text {th }} 2021$, and we expect to complete recruitment of subjects within one and half year from registration date (5-7-2022). The current protocol version is 1.1, date: 11-10-2019.

Acknowledgements

Not applicable

\section{Funding}

The study is funded by The fund to support independent, strategic clinical research in the Region of Southern Denmark (Region Syddanmarks pulje for fri og strategisk Forskning), The fund to support clinical doctoral candidates in the Region of Southern Denmark (Region Syddanmarks pulje til støtte af Ph.d.-forløb), The A.P. Møller Foundation, The Department of Regional Health Research at University of Southern Denmark and Steno Diabetes Center Odense, Denmark, which is funded by the Novo Nordisk Foundation. The funding parties have no influence on the study design, study conduct, results, or dissemination. 
Availability of data and materials

The trial dataset is accessed by the investigators (SG and SGH). SG will perform the data analysis and takes responsibility for the integrity of the data. There are no results available from this study protocol. The public can access the data upon reasonable request.

Authors' contributions

BG, CBJ, PH and SGH: idea conception, original protocol, statistical plan, sample size, and design. SG and SGH: revision of the protocol and endpoint design. SG and SGH: trial management, protocol amendments, updates, and protocol paper draft. SG: writing the first draft of the manuscript and editing. All authors reviewed and commented on drafts of the paper and approved the final version.

Ethics approval and consent to participate

The Regional Committees on Health Research Ethics for Southern Denmark approved the study (project identifier S-20190134) and all subjects provided informed consent.

Consent for publication

Not applicable

Competing interests

The investigators have no competing interests in the study.

Publisher's Note

Author details

SG: Søren Gam, MSc, Department of Medicine, University hospital of Southern Denmark and Steno Diabetes Center, Odense, Denmark.

BG: Bibi Gram, MSc, PhD, Associate Professor, The Research Unit of Health Sciences, University hospital of Southern Denmark.

CBJ: Claus Bogh Juhl, MD, PhD, Clinical Professor. Department of Medicine, University hospital of Southern Denmark and Steno Diabetes Center, Odense, Denmark.

PH: Pernille Hermann, MD, PhD, Associate Professor, Department of Endocrinology, Odense University Hospital, Odense, Denmark.

SGH: Stinus Gadegaard Hansen, MD, PhD, Associate Professor, Department of Medicine, University hospital of Southern Denmark and Department of Regional Health Research. 


\section{Abbreviations}

2MWT

2 minute walk test

aBMD

Areal bone mineral densit

BMD

Bone mineral density

BMI

Body mass index

BMSi

Bone Material Strength index

$\mathrm{BV} / \mathrm{TV}$

Bone volume per trabecular volume

CTX-1

Carboxy-terminal type-1 collagen

DXA

Whole-body dual-energy X-ray absorptiometry

GIP

Gastric inhibitory protein

GLP 1

Glucagon-like-peptide 1

GLP 2

Glucagon-like-peptide 2

HR-pQCT

High-resolution peripheral quantitative computed tomography

IPAQ

International Physical Activity Questionnaire

P1NP

Procollagen type-1 amino-terminal propeptide

QCT

Quantitative computed tomography

RYGB

Roux-en-Y-gastric bypass

SAE

Serious adverse events

SAR

Serious adverse reactions

SG

Sleeve gastrectomy 
SPIRIT

Standard Protocol Items Recommendations for Interventional Trials

SPPB

Short Physical Performance Battery

T25FWT

Timed 25-ft walk test

vBMD

Volumetric bone mineral density

\section{References}

1. Ng M, Fleming T, Robinson M, Thomson B, Graetz N, Margono C, et al. Global, regional, and national prevalence of overweight and obesity in children and adults during 1980-2013: a systematic analysis for the Global Burden of Disease Study 2013. Lancet. 2014;384(9945):766-81.

2. Arterburn D, Gupta A. Comparing the Outcomes of Sleeve Gastrectomy and Roux-en-Y Gastric Bypass for Severe Obesity. JAMA. 2018;319(3):235-7.

3. Lindeman KG, Greenblatt LB, Rourke C, Bouxsein ML, Finkelstein JS, Yu EW. Longitudinal 5-Year Evaluation of Bone Density and Microarchitecture After Roux-en-Y Gastric Bypass Surgery. J Clin Endocrinol Metab. 2018;103(11):4104-12.

4. Mundbjerg LH, Stolberg CR, Bladbjerg EM, Funch-Jensen P, Juhl CB, Gram B. Effects of 6 months supervised physical training on muscle strength and aerobic capacity in patients undergoing Rouxen-Y gastric bypass surgery: a randomized controlled trial. Clin Obes. 2018;8(4):227-35.

5. Muschitz C, Kocijan R, Haschka J, Zendeli A, Pirker T, Geiger C, et al. The Impact of Vitamin D, Calcium, Protein Supplementation, and Physical Exercise on Bone Metabolism After Bariatric Surgery: The BABS Study. J Bone Miner Res. 2016;31(3):672-82.

6. Shanbhogue VV, Støving RK, Frederiksen KH, Hanson S, Brixen K, Gram J, et al. Bone structural changes after gastric bypass surgery evaluated by HR-pQCT: a two-year longitudinal study. Eur $\mathrm{J}$ Endocrinol. 2017;176(6):685-93.

7. Yu EW. Bone metabolism after bariatric surgery. J Bone Miner Res. 2014;29(7):1507-18.

8. Lu CW, Chang YK, Chang HH, Kuo CS, Huang CT, Hsu CC, et al. Fracture Risk After Bariatric Surgery: A 12-Year Nationwide Cohort Study. Med (Baltim). 2015;94(48):e2087.

9. Nakamura KM, Haglind EG, Clowes JA, Achenbach SJ, Atkinson EJ, Melton LJ. 3rd, et al. Fracture risk following bariatric surgery: a population-based study. Osteoporos Int. 2014;25(1):151-8.

10. Rousseau C, Jean S, Gamache P, Lebel S, Mac-Way F, Biertho L, et al. Change in fracture risk and fracture pattern after bariatric surgery: nested case-control study. BMJ. 2016;354:i3794.

11. Yu EW, Lee MP, Landon JE, Lindeman KG, Kim SC. Fracture Risk After Bariatric Surgery: Roux-en-Y Gastric Bypass Versus Adjustable Gastric Banding. J Bone Miner Res. 2017;32(6):1229-36. 
12. Axelsson KF, Werling M, Eliasson B, Szabo E, Näslund I, Wedel H, et al. Fracture Risk After Gastric Bypass Surgery: A Retrospective Cohort Study. J Bone Miner Res. 2018;33(12):2122-31.

13. Gagnon C, Schafer AL. Bone Health After Bariatric Surgery. JBMR Plus. 2018;2(3):121-33.

14. Schafer AL, Weaver CM, Black DM, Wheeler AL, Chang H, Szefc GV, et al. Intestinal Calcium Absorption Decreases Dramatically After Gastric Bypass Surgery Despite Optimization of Vitamin D Status. J Bone Miner Res. 2015;30(8):1377-85.

15. Oppert JM, Bellicha A, Roda C, Bouillot JL, Torcivia A, Clement K, et al. Resistance Training and Protein Supplementation Increase Strength After Bariatric Surgery: A Randomized Controlled Trial. Obesity (Silver Spring). 2018;26(11):1709-20.

16. Black DM, Delmas PD, Eastell R, Reid IR, Boonen S, Cauley JA, et al. Once-yearly zoledronic acid for treatment of postmenopausal osteoporosis. N Engl J Med. 2007;356(18):1809-22.

17. Boonen S, Reginster JY, Kaufman JM, Lippuner K, Zanchetta J, Langdahl B, et al. Fracture risk and zoledronic acid therapy in men with osteoporosis. N Engl J Med. 2012;367(18):1714-23.

18. Deas CM, Murphy P, Iranikhah M, Freeman MK. Retained Skeletal Effects of Zoledronic Acid Following Discontinuation of Treatment: A Review of the Literature. Consult Pharm. 2017;32(3):14455.

19. Liu Y, Côté MM, Cheney MC, Lindeman KG, Rushin CC, Hutter MM, et al. Zoledronic acid for prevention of bone loss in patients receiving bariatric surgery. Bone Rep. 2021;14:100760.

20. Børsheim E, Herndon DN, Hawkins HK, Suman OE, Cotter M, Klein GL. Pamidronate attenuates muscle loss after pediatric burn injury. J Bone Miner Res. 2014;29(6):1369-72.

21. Harada A, Ito S, Matsui Y, Sakai Y, Takemura M, Tokuda H, et al. Effect of alendronate on muscle mass: Investigation in patients with osteoporosis. Osteoporosis Sarcopenia. 2015;1(1):53-8.

22. Watanabe R, Fujita N, Takeda S, Sato Y, Kobayashi T, Morita M, et al. Ibandronate concomitantly blocks immobilization-induced bone and muscle atrophy. Biochem Biophys Res Commun. 2016;480(4):662-8.

23. Miedany YE, Gaafary ME, Toth M, Hegazi MO, Aroussy NE, Hassan W, et al. Is there a potential dual effect of denosumab for treatment of osteoporosis and sarcopenia? Clin Rheumatol. 2021.

24. Park JH, Park KH, Cho S, Choi YS, Seo SK, Lee BS, et al. Concomitant increase in muscle strength and bone mineral density with decreasing IL-6 levels after combination therapy with alendronate and calcitriol in postmenopausal women. Menopause. 2013;20(7):747-53.

25. Klein GL. The Role of Bone in Muscle Wasting. Int J Mol Sci. 2020;22(1).

26. Nevitt MC, Thompson DE, Black DM, Rubin SR, Ensrud K, Yates AJ, et al. Effect of alendronate on limited-activity days and bed-disability days caused by back pain in postmenopausal women with existing vertebral fractures. Fracture Intervention Trial Research Group. Arch Intern Med. 2000;160(1):77-85.

27. Chan AW, Tetzlaff JM, Altman DG, Laupacis A, Gøtzsche PC, Krleža-Jerić K, et al. SPIRIT 2013 statement: defining standard protocol items for clinical trials. Ann Intern Med. 2013;158(3):200-7. 
28. Sealed_Envelope_Ltd. Create a blocked randomisation list 2021 [Available from: https://www.sealedenvelope.com/simple-randomiser/v1/lists.

29. Hart NH, Newton RU, Tan J, Rantalainen T, Chivers P, Siafarikas A, et al. Biological basis of bone strength: anatomy, physiology and measurement. J Musculoskelet Neuronal Interact. 2020;20(3):347-71.

30. Boutroy S, Bouxsein ML, Munoz F, Delmas PD. In vivo assessment of trabecular bone microarchitecture by high-resolution peripheral quantitative computed tomography. J Clin Endocrinol Metab. 2005;90(12):6508-15.

31. Burghardt AJ, Buie HR, Laib A, Majumdar S, Boyd SK. Reproducibility of direct quantitative measures of cortical bone microarchitecture of the distal radius and tibia by HR-pQCT. Bone. 2010;47(3):51928.

32. Pistoia W, van Rietbergen B, Lochmüller EM, Lill CA, Eckstein F, Rüegsegger P. Image-based microfinite-element modeling for improved distal radius strength diagnosis: moving from bench to bedside. J Clin Densitom. 2004;7(2):153-60.

33. Diez-Perez A, Bouxsein ML, Eriksen EF, Khosla S, Nyman JS, Papapoulos S, et al. Technical note: Recommendations for a standard procedure to assess cortical bone at the tissue-level in vivo using impact microindentation. Bone Rep. 2016;5:181-5.

34. Fess. Clinical assessment recommendations. 1st ed. Indianapolis ed. Indianapolis: American Society of Hand Therapists; 1981.

35. Freire AN, Guerra RO, Alvarado B, Guralnik JM, Zunzunegui MV. Validity and reliability of the short physical performance battery in two diverse older adult populations in Quebec and Brazil. J Aging Health. 2012;24(5):863-78.

36. Ekelund U, Sepp H, Brage S, Becker W, Jakes R, Hennings M, et al. Criterion-related validity of the last 7-day, short form of the International Physical Activity Questionnaire in Swedish adults. Public Health Nutr. 2006;9(2):258-65.

37. Harris PA, Taylor R, Thielke R, Payne J, Gonzalez N, Conde JG. Research electronic data capture (REDCap)-a metadata-driven methodology and workflow process for providing translational research informatics support. J Biomed Inform. 2009;42(2):377-81.

38. Campanha-Versiani L, Pereira DAG, Ribeiro-Samora GA, Ramos AV, de Sander Diniz MFH, De Marco LA, et al. The Effect of a Muscle Weight-Bearing and Aerobic Exercise Program on the Body Composition, Muscular Strength, Biochemical Markers, and Bone Mass of Obese Patients Who Have Undergone Gastric Bypass Surgery. Obes Surg. 2017;27(8):2129-37.

39. Reid IR, Horne AM, Mihov B, Stewart A, Garratt E, Wong S, et al. Fracture Prevention with Zoledronate in Older Women with Osteopenia. N Engl J Med. 2018;379(25):2407-16.

40. Andersen S, Frederiksen KD, Hansen S, Brixen K, Gram J, Støving RK. Bone structure and estimated bone strength in obese patients evaluated by high-resolution peripheral quantitative computed tomography. Calcif Tissue Int. 2014;95(1):19-28. 
41. Sornay-Rendu E, Boutroy S, Vilayphiou N, Claustrat B, Chapurlat RD. In obese postmenopausal women, bone microarchitecture and strength are not commensurate to greater body weight: the Os des Femmes de Lyon (OFELY) study. J Bone Miner Res. 2013;28(7):1679-87.

42. Compston JE, Watts NB, Chapurlat R, Cooper C, Boonen S, Greenspan S, et al. Obesity is not protective against fracture in postmenopausal women: GLOW. Am J Med. 2011;124(11):1043-50.

43. Prieto-Alhambra D, Premaor MO, Fina Avilés F, Hermosilla E, Martinez-Laguna D, Carbonell-Abella C, et al. The association between fracture and obesity is site-dependent: a population-based study in postmenopausal women. J Bone Miner Res. 2012;27(2):294-300.

44. Bredella MA, Greenblatt LB, Eajazi A, Torriani M, Yu EW. Effects of Roux-en-Y gastric bypass and sleeve gastrectomy on bone mineral density and marrow adipose tissue. Bone. 2017;95:85-90.

45. Brzozowska MM, Tran T, Bliuc D, Jorgensen J, Talbot M, Fenton-Lee D, et al. Roux-en-Y gastric bypass and gastric sleeve surgery result in long term bone loss. Int J Obes (Lond). 2021;45(1):23546.

46. Cadart O, Degrandi O, Barnetche T, Mehsen-Cetre N, Monsaingeon-Henry M, Pupier E, et al. Long-Term Effects of Roux-en-Y Gastric Bypass and Sleeve Gastrectomy on Bone Mineral Density: a 4-Year Longitudinal Study. Obes Surg. 2020;30(9):3317-25.

47. Hart NH, Nimphius S, Rantalainen T, Ireland A, Siafarikas A, Newton RU. Mechanical basis of bone strength: influence of bone material, bone structure and muscle action. J Musculoskelet Neuronal Interact. 2017;17(3):114-39.

48. Sylvia LG, Bernstein EE, Hubbard JL, Keating L, Anderson EJ. Practical guide to measuring physical activity. J Acad Nutr Diet. 2014;114(2):199-208.

\section{Figures}




\begin{tabular}{|c|c|c|c|c|c|}
\hline $\begin{array}{c}\text { Enrolment } \\
\text { (Month }-8 \text { to Month }-1 \text { ) }\end{array}$ & $\begin{array}{c}\text { Baseline } \\
\text { (Month -3 to Week -2) }\end{array}$ & $\begin{array}{c}\text { Intervention } \\
\text { (Days }-59 \text { to Days }-7)\end{array}$ & $\begin{array}{c}\text { Surgery } \\
\text { (Day 0) }\end{array}$ & $\begin{array}{l}\text { Follow-up } \\
\text { (Month 12) }\end{array}$ & $\begin{array}{l}\text { Follow-up } \\
\text { (Month 24) }\end{array}$ \\
\hline $\begin{array}{l}\text { Screening } \\
\text { Information } \\
\text { Enrollment } \\
\text { Written informed } \\
\text { consent }\end{array}$ & $\begin{array}{l}\text { Assessments } \\
\text { - QCT } \\
\text { - DXA } \\
\text { - HRpQCT } \\
\text { - Microindentation } \\
\text { - Blood samples } \\
\text { - Muscle strength } \\
\text { - Physical function } \\
\text { - Physical activity } \\
\text { Randomization }\end{array}$ & $\begin{array}{c}\begin{array}{c}\text { Zoledronic } \\
\text { Acid }\end{array} \\
\text { Placebo }\end{array}$ & $\begin{array}{c}\text { Roux-en-Y } \\
\text { gastric bypass } \\
\text { or } \\
\text { Sleeve } \\
\text { gastrectomy }\end{array}$ & $\begin{array}{l}\text { Assessments } \\
\text { - QCT } \\
\text { - DXA } \\
\text { - HRpQCT } \\
\text { - Microindentation } \\
\text { - Blood samples } \\
\text { - Muscle strength } \\
\text { - Physical function } \\
\text { - Physical activity }\end{array}$ & $\begin{array}{l}\text { Assessments } \\
\text { - QCT } \\
\text { - DXA } \\
\text { - HRpQCT } \\
\text { - Microindentation } \\
\text { - Blood samples } \\
\text { - Muscle strength } \\
\text { - Physical function } \\
\text { - Physical activity }\end{array}$ \\
\hline
\end{tabular}

\section{Figure 1}

shows the study design. QCT: Quantitative Computed Tomography; DXA: whole-body dual-energy X-ray absorptiometry; HR-pQCT: high-resolution peripheral quantitative computed tomography

\section{Supplementary Files}

This is a list of supplementary files associated with this preprint. Click to download.

- 21.12.10SPIRITFillablechecklistFINAL.doc 